\title{
Magnetic field calculations within substation environment for EMC studies
}

\author{
A. I. Tarmizi \\ University of Southampton, UK \\ Universiti Teknikal Malaysia Melaka \\ ait1g12@soton.ac.uk
}

\author{
Mihai D. Rotaru \\ University of Southampton, UK \\ mr@ecs.soton.ac.uk
}

\author{
Jan K. Sykulski \\ University of Southampton, UK \\ jks@soton.ac.uk
}

\begin{abstract}
The electromagnetic environment within high voltage substations needs to be correctly predicted and quantified especially as more and more sensitive microelectronic devices are introduced in proximity to the switching devices in the switchyard. This trend will only increase with the advent of the 'smart grid'; therefore there is a need to re-evaluate the substation environment for EMC assessment, accounting for new scenarios. In this paper, the magnetic field distribution within a substation is calculated using an in-house developed numerical algorithm within Matlab. The flow of current in the substation is used as an input to the code and fields within the substation are then evaluated. The reported code can calculate the magnetic field distribution within an air insulated (AIS) high voltage substation under normal operating conditions as well as for the case of a lightning strike situation.
\end{abstract}

Keywords- EMC; magnetic field; Matlab; Digsilent PowerFactory; lightning current;

\section{INTRODUCTION}

The ability of an equipment or system to function satisfactorily in its electromagnetic environment without introducing intolerable electromagnetic disturbances to anything in that environment is known as Electromagnetic Compatibility (EMC) [1]. In a high voltage substation, while the components and equipment are operating, electric and magnetic fields are produced that might or might not cause interruption to the system; thus it is important to make sure that the system is compatible and immune to electromagnetic interferences $[2,3]$.

EMC in power systems is a topic which has been widely covered in literature. However, there has been limited work done on the EMC within an automated substation, especially in the context of a smart grid implementation. Substation automation is a term applied to an electrical substation that manages the operation between distributed intelligent electronic devices (IEDs) interconnected by communication networks [4]. This implies that microelectronic devices, such as microcontrollers and microprocessors, which provide the 'intelligence', are being installed closer to high voltage and high power switching devices within the switchyard of high voltage substations. In this context, it is important that the electromagnetic field distribution within a substation is well understood so that possible EMC hazards are minimised. Measurements and/or simulations can be employed to build a picture of the field distribution within the area of interest. Deciding on which method to use depends on many factors; however, in most of the published work, both approaches are used [5-8]. Usually computational methods are used to test cases that are difficult or impossible to be measured, such as lightning strikes; on the other hand, numerical methods are normally validated against a set of measurements [5].

Under normal operating conditions a high voltage substation system produces electric and magnetic fields at low frequency, $50 \mathrm{~Hz}$ or $60 \mathrm{~Hz}$. However, high frequency fields can also be generated due to disturbances that occur in the system, such as switching and lightning strikes. According to [9] frequencies up to $30 \mathrm{GHz}$ can be generated by disturbances occurring naturally or due to man-made sources.

From a health and safety point of view, these generated fields should be within the range of health regulations and EMC standards, with different countries setting their own national standards. The majority of the national guidelines are set by the International Commission on Non-Ionizing Radiation Protection (ICNIRP) which is recognized by the World Health Organization (WHO) as a non-governmental organization that evaluates scientific results from all over the world. The limits recommended by the ICNIPR and WHO are summarized in Table I [10].

TABLE I. ICNIRP EXPOSURE GUIDELINES AT POWER FREQUENCY 50HZ

\begin{tabular}{|l|c|c|}
\hline & $\begin{array}{c}\text { Magnetic field } \\
\text { intensity, } \mathbf{H}(\mathbf{A} / \mathbf{m})\end{array}$ & $\begin{array}{c}\text { Magnetic flux } \\
\text { density, B ( } \boldsymbol{\mu T})\end{array}$ \\
\hline Public exposure limits & 80 & 100 \\
\hline Occupational exposure limits & 400 & 500 \\
\hline
\end{tabular}

\section{Calculation of Magnetic Fields within a Substation}

Under normal operating conditions magnetic and electric fields exist around the current carrying equipment within the substation. It is expected that the highest magnitude of the fields will be observed around the paths that carry the highest currents. However, there are various factors affecting the level of the fields, such as current magnitude, phase spacing, bus height, phase configuration, distance from the source and the phase unbalance. It is rather difficult to predict the distribution of magnetic field in the substation environment using popular numerical methods, such as finite differences and finite elements, mainly because of the large size of the electromagnetic problem to be solved; these methods will struggle with meshing large domains such as the whole substation environment. We have employed directly the Biot-Savart Law because of its simplicity and computational efficiency. Obviously all components and equipment structure and dimensions need to be included. 
According to the Biot-Savart Law the magnetic field due to a current element of length $d l$ is given by [11]

$$
\bar{H}=\frac{I}{4 \pi} \int_{l} \frac{\overline{d l} \times \bar{R}}{R^{2}}
$$

A simple MATLAB routine was developed to calculate the field in three dimensions (3D) within the air environment. The total magnetic field intensity, $H$, at each position $(x, y, z)$ and at any instant of time $t$ is then given by

$$
\begin{gathered}
H(x, y, z, t)=\hat{x} H_{x}(t)+\hat{y} H_{y}(t)+\hat{z} H_{z}(t) \\
|H|=\sqrt{H_{x}^{2}+H_{y}^{2}+H_{z}^{2}}
\end{gathered}
$$

with superposition applied at every location. To illustrate the approach a particular substation has been considered.

\section{A. Substation structure and modelling assumptions}

For this work, the layout of the Rosiori substation was provided by the Technical University of Cluj, Faculty of Electrotechnics. The substation consists of a $400 \mathrm{kV}$ double busbar with three outgoing feeders that connect to three loads and a shunt reactor which is a compensation coil. The autotransformer then steps down the voltage to $220 \mathrm{kV}$ and finally there are three outgoing feeders on the $220 \mathrm{kV}$ side [12]. One of the reasons that this particular substation was chosen was the availability of magnetic and electric field measurements [7] which will be used to validate the developed calculation model.

In order to make the modelling tool efficient we decided to focus on the main sources of the magnetic field and thus neglect the less important influences. The first simplification was to include only the conductors above the ground, with the substation busbars treated effectively as transmission lines and currents and voltages of each branch computed. All other equipment, as well as the insulators, are neglected and thus only the magnetic field produced by the conductors in the substation is considered. Although there are some differences in the results when compared with the case where more details are accounted for, as discussed in [13], the errors are relatively minor and hence the simplification is considered acceptable. In fact the main difference observed and reported in [12] is not in the overall field distribution but rather in the finer details of the results, such as the closeness of the maximum field to the substation equipment. If the field is to be calculated far enough from the current paths the assumption used here is justified.

Another important substation component which would normally be considered is the transformer. The transformers installed in substations, however, are usually very well shielded, so that most of their magnetic field is confined within their casing; consequently, their contribution to the magnetic field of the outer environment is relatively small. It would be very expensive computationally to simulate the whole transformer [14], therefore - for this particular research study - only the connections between the transformer terminals and the cables are considered. This simplification appears to be fully justified for the normal operation of the system, but may need to be reconsidered in the case of a lightning strike situation, especially when the strike is close to the transformer.

\section{B. Modelling procedures}

The algorithm consists of two main steps. First, the geometry (and topology) of the substation is introduced to identify all conducting paths. Each path is then divided into sub-segments in the $x y z$ coordinate system and currents are applied to each conductor. This information would usually come from a power flow solution provided by appropriate modeller (such as PowerFactory [15]) for normal operating conditions. The BiotSavart Law is then applied to calculate the magnetic field generated by the applied currents. For the illustrative example reported in this paper, a plane grid of 6674 calculation points is arranged for the substation environment for magnetic field calculations. Along the $x$ axis the increment is a $3 \mathrm{~m}$ interval $(x+3)$ and for the $y$ axis it is a $2 \mathrm{~m}$ interval $(y+2)$ as illustrated in Fig. 1. With this algorithm the density of the calculation points can be easily modified to focus on regions of higher interest.

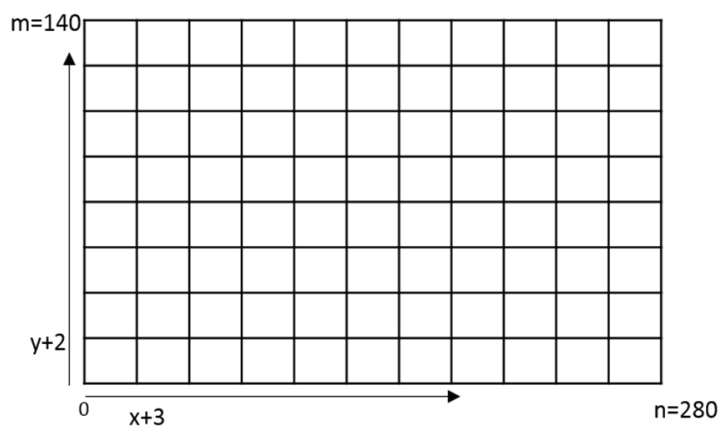

Fig. 1. A grid of points for magnetic field calculations for a substation.

The substation considered is $280 \mathrm{~m}$ long, $140 \mathrm{~m}$ wide and the conductors are located at the height of $12 \mathrm{~m}$ above the ground (on the $400 \mathrm{kV}$ side). For such a large site, it takes almost 6 hours for a computer with Intel (R) Core (TM) $3.4 \mathrm{GHz}$ processor with $16 \mathrm{~GB}$ of RAM to complete the magnetic field calculations for a matrix of points $94 \times 71$ in $x$ and $y$ axes, respectively. The generated magnetic field is studied at different levels from the ground by setting the height for $z$ axis in the algorithm. In [16], the magnetic field was measured at three different heights based on the human body measurements, namely: the feet at $0 \mathrm{~m}$, the waist at $1 \mathrm{~m}$ and the head at $1.75 \mathrm{~m}$. The computing times will obviously depend on the number of current inputs.

Two particular cases of magnetic field calculation have been considered. For the first case a normal distribution of three phase currents is assumed resulting from a load flow analysis. The second case introduces a lightning strike and associated current injected into substation conductors. Thus both the steady state and a particular disturbance have been considered. Although for the case of the lighting strike the assumptions introduced earlier on may be a little too restrictive, the results will nevertheless demonstrate the capability of the algorithm to cope with transient as well as steady-state currents. To show this capability a frequency domain analysis was also implemented and the results in terms of magnetic field intensity are presented and compared with the time domain analysis. 


\section{Magnetic Field Computational Results}

\section{A. Nominal input current}

The Rosiori substation has the $400 \mathrm{kV}$ side connected to three loads, a shunt reactance and an auto transformer to step down the voltage to $220 \mathrm{kV}$. The normal operating currents at frequency of $50 \mathrm{~Hz}$ for each load, as listed in Table II, are used for the magnetic field calculations.

TABLE II. OPERATING CURRENTS OF THE ROSIORI STATION 400KV SIDE

\begin{tabular}{|l|c|}
\hline Load Component & Operating current (A) \\
\hline Load 1 (Mukacevo) & 200 \\
\hline Load 2 (Oradea) & 90 \\
\hline Load 3 (Gadalin) & 28 \\
\hline Shunt Reactance & 147 \\
\hline
\end{tabular}

The magnetic field distribution can be evaluated at different heights from the ground, but for this particular substation this was calculated and plotted only at the height of $1.7 \mathrm{~m}$ since the measurements were done at this height [6]. The predicted and experimental distributions will be compared in the context of the exposure limits.

The computed results for the normal operating currents are presented in Fig. 2. It shows that the predicted highest value of the magnetic field is $4.164 \mathrm{~A} / \mathrm{m}$ located along busbar 1 , where it is connected to Load 1 with 200A. The measurements were reported in [5] and it is worth mentioning that the distribution of the magnetic field was obtained through more than 3000 test points scattered around the whole area of the substation. The meter was placed at $1.7 \mathrm{~m}$ above the ground. The measurements were performed under normal working conditions and by monitoring the current flow in all bays. The highest magnetic field measured was $4 \mathrm{~A} / \mathrm{m}$, thus the prediction is within $3.5 \%$ of the measured maximum, while both values are within the public exposure limits. The good agreement between the modelling and experiment increases confidence in the results and provides some justification for the assumptions made.

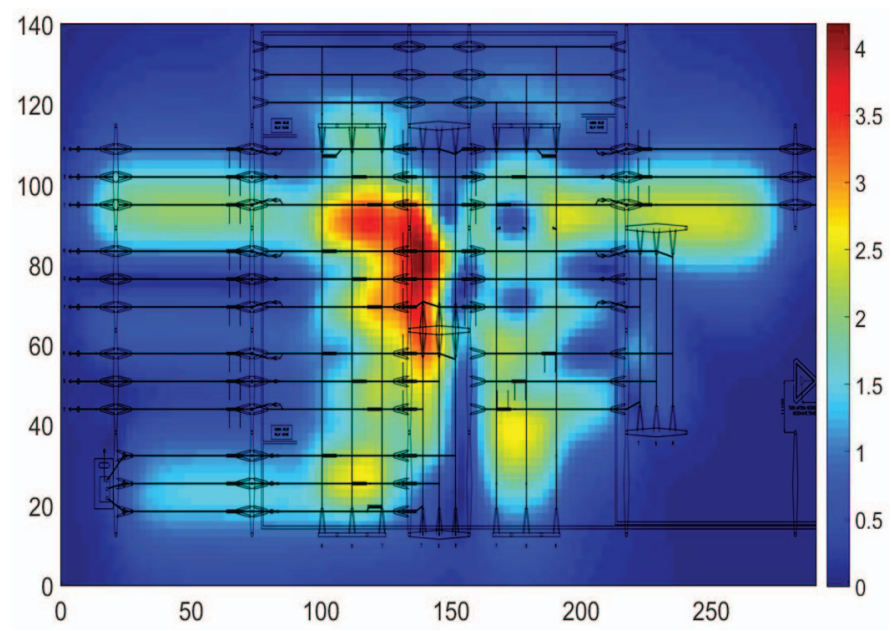

Fig. 2. The computed magnetic field distribution (in $\mathrm{A} / \mathrm{m}$ ) at the Rosiori substation at the height of $1.7 \mathrm{~m}$ above the ground.

\section{B. Input current with a single lightning pulse}

In the second test, a single 200kA IEC62305-1 [17] lightning pulse with $19 / 459 \mu$ s front and tail times was injected as a disturbance in the substation circuit as illustrated in Fig. 3.

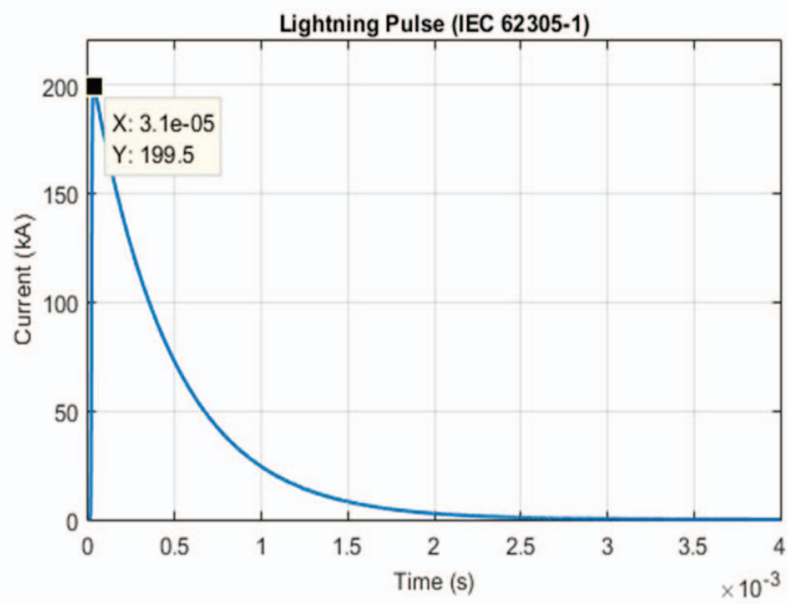

Fig. 3. IEC62305-1 lightning pulse injected to the substation.

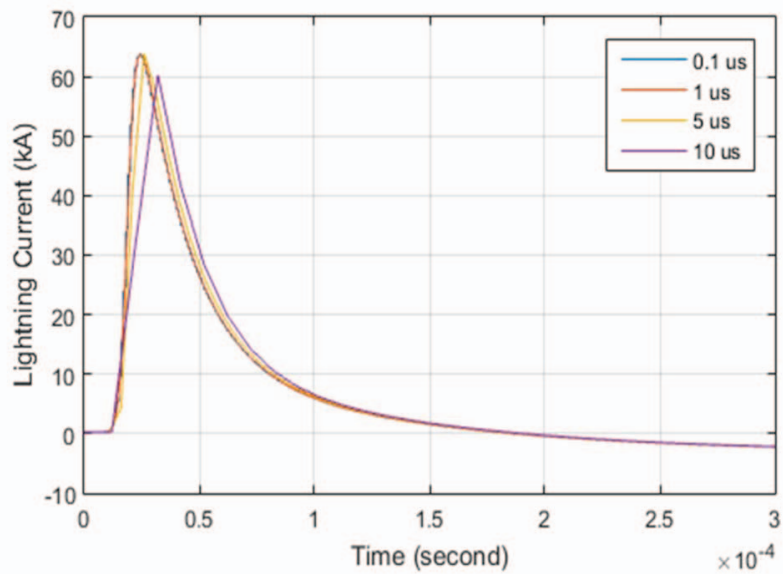

Fig. 4. Lightning current at one of the load (Mukachevo) for four different time steps.

Using the Power Factory software [15], the load flow in the substation was supplemented by an addition of a lightning pulse injected at $0 \mathrm{~s}$ at one of the $400 \mathrm{kV}$ busbars. The time step of this transient analysis is one of the important parameters that needs to be correctly set to achieve meaningful results. The time step will influence the length of the simulation so it is important that it is not set to a very small value. To decide on a suitable time step value, several simulations with different time step were set up and run. The results in terms of the currents in one of the loads (Mukachevo) are shown in Fig. 4 for four different time steps: $0.1 \mu \mathrm{s}, 1 \mu \mathrm{s}, 5 \mu \mathrm{s}$, and $10 \mu \mathrm{s}$, respectively. It is clear that a $10 \mu \mathrm{s}$ time step is not sufficient and the peak current calculated using $5 \mu \mathrm{s}$ and $1 \mu \mathrm{s}$ steps is about 5\% larger; moreover, the time at which the maximum current is reached is also different. When the time step was further reduced to $0.1 \mu \mathrm{s}$ no major differences were observed compared with the $1 \mu$ s case. Consequently, for the simulations presented here, a $1 \mu$ s time step was used. 
The currents of each load with the lightning pulse were used as an input to predict the magnetic field distributions (Fig. 5). The highest current magnitude in the system at $0.02 \mathrm{~s}$ was $64 \mathrm{kA}$ at Mukacevo. The charge conservation is enforced within the model by making sure that the total currents flowing in and out of the substation sum up to zero.

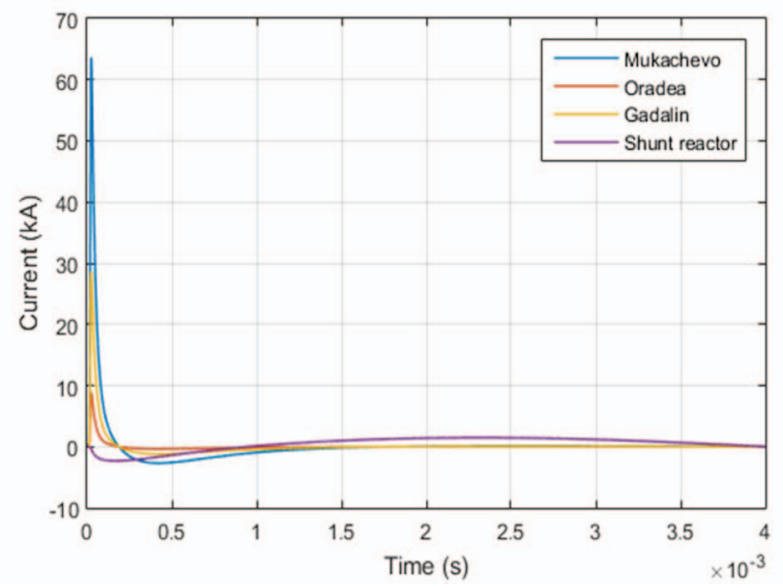

Fig. 5. The current waveforms when a lightning pulse is added.

The magnetic field distribution in the substation due to the lightning pulse can be considered in two different ways, in time domain and in frequency domain. Both approaches have been applied and compared.

\section{1) Magnetic field calculation: the time domain approach}

The current flow with a lightning pulse added varies in time as illustrated in Fig. 5, thus the magnetic field changes with time too. Since the lightning current is changing fast, the current element needs to be small enough to capture this fast transient situation; however, it has been found that a $1 \mathrm{~m}$ long element is sufficient to yield good precision of the result. The time step used for this simulation was $1 \mu \mathrm{s}$ and the total time of the simulation 0.0003s. The highest magnetic field is plotted in Fig. 6 and is obtained at the time of $24 \mu \mathrm{s}$. The field is plotted at $1.75 \mathrm{~m}$ from the ground and the maximum is $1282 \mathrm{~A} / \mathrm{m}$.

The magnetic field calculated shows that the maximum magnetic field value exceeds the public and the occupational exposure limit set by ICNIRP. The calculated magnetic field then could be used for further electromagnetic compatibility analysis. The field may be harmful to the equipment in the proximity of the hot spots shown in Fig. 6.

\section{2) Magnetic field calculation: the frequency domain approach}

In order to calculate the magnetic field in the frequency domain, Prony Analysis and Fast Fourier Transform (FFT), the frequency analysis tools from the Digsilent Power Factory, were used. Prony Analysis was used to decompose the signal into damped sinusoidal oscillations to determine the exact value of the important harmonic signals over a range of frequencies [15]. These results compare well with the existent data in the public domain that the induced currents due to the lightning strike have the frequency ranging from $100 \mathrm{kHz}$ to $120 \mathrm{MHz}$ [18]. The magnitude and frequencies decomposed from each three phase load currents using Prony Analysis are shown in Fig. 7. The important frequencies that were used in the magnetic field calculations are: $f_{1}$, the fundamental frequency at $50 \mathrm{~Hz}, \mathrm{f}_{2}$ at $70 \mathrm{kHz}, \mathrm{f}_{3}$ at $100 \mathrm{kHz}, \mathrm{f}_{4}$ at $150 \mathrm{kHz}, \mathrm{f}_{5}$ at $200 \mathrm{kHz}$, and lastly $\mathrm{f}_{6}$ at $250 \mathrm{kHz}$.

The model was then excited separately by each of these harmonics with their respective amplitude. The magnetic field for each frequency was then computed. Finally, the results were combined by adding all the field results in terms of $x, y$ and $z$ components from all the harmonics. A typical result is shown in Figs. 8 and 9.

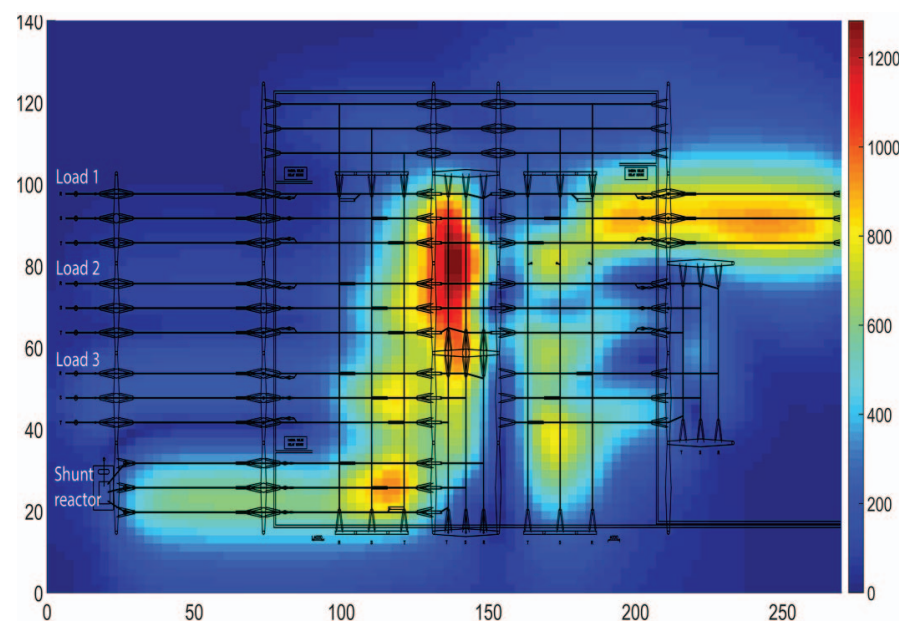

Fig. 6. Distribution of the magnetic field (in $\mathrm{A} / \mathrm{m}$ ) due to a lightning strike in time domain.
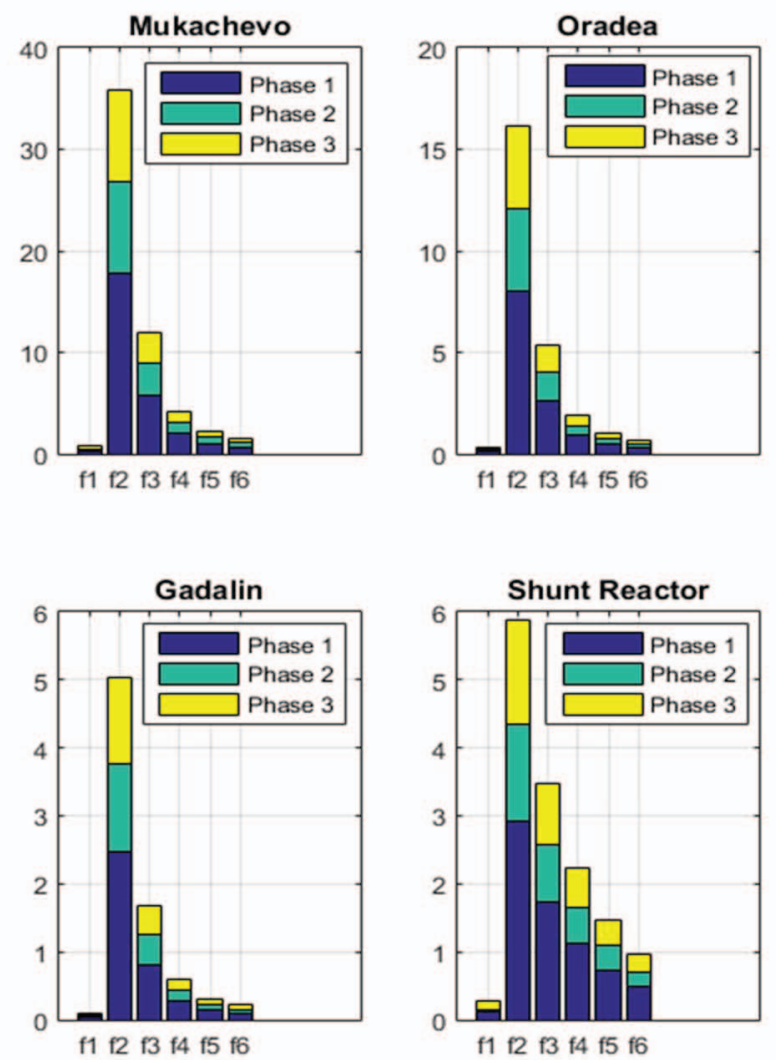

Fig. 7. The important harmonics of each three phase current at every load. 


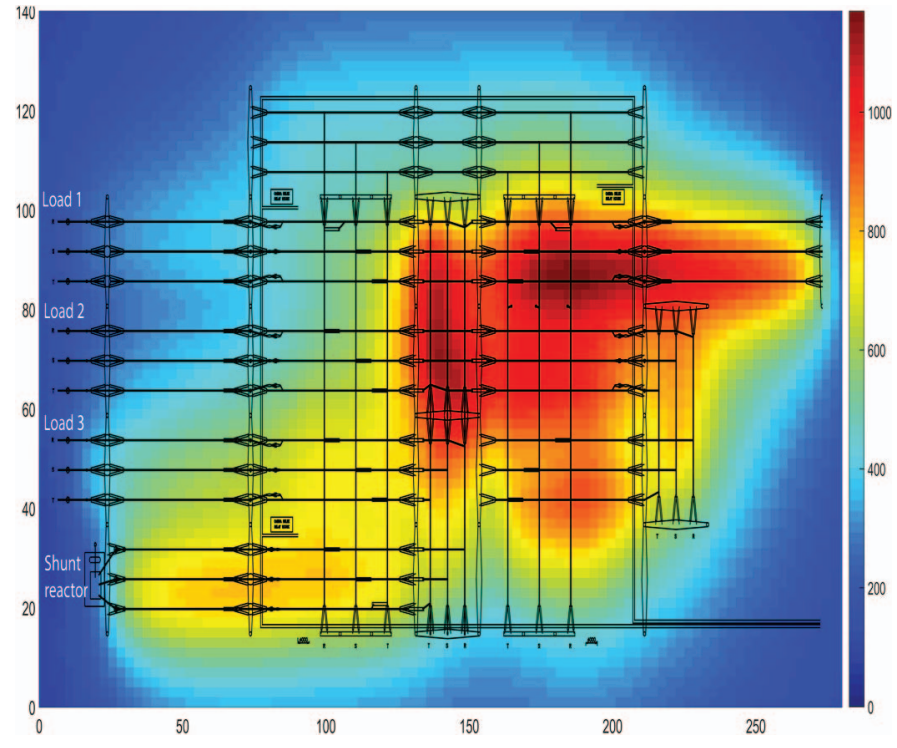

Fig. 8. Distribution of the magnetic field (in $\mathrm{A} / \mathrm{m}$ ) due to a lightning strike calculated using the frequency domain aproach

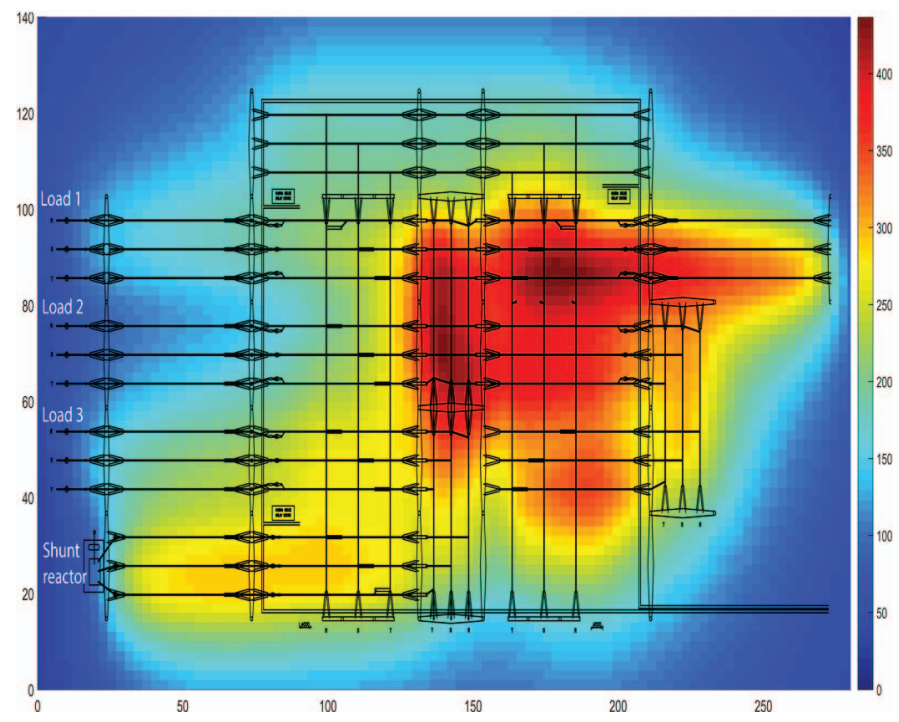

Fig. 9. Distribution of the magnetic field (in $\mathrm{A} / \mathrm{m}$ ) in frequency domain without a $70 \mathrm{kHz}$ harmonic.

The highest magnetic field computed using this approach is $1166 \mathrm{~A} / \mathrm{m}$, this value being $9.04 \%$ smaller than the value obtained from the time domain analysis. The difference can be attributed to the fact that we are only considering six harmonics whereas in the time domain all the harmonics carrying power are accounted for. However, the approach allows us to separate the effects of each of these harmonics. For example, in Fig. 9 the magnetic field intensity plot without the $70 \mathrm{kHz}$ harmonic is presented: the maximum magnetic field only reaches $436.4 \mathrm{~A} / \mathrm{m}$. Therefore if the $70 \mathrm{kHz}$ harmonic were to be filtered out a reduction of more than half could be achieved. This suggests that to protect sensitive electronic equipment in the substation environment an appropriate filter may be sufficient.

Both analyses, the time domain and frequency domain, predict the hot spot in the same location, which gives confidence in the proposed methodology and the computational tool. The hot spot for the Rosiori substation, studied here, is close to the connection towards the autotransformer. As demonstrated, this relatively simple approach allows the calculation of the magnetic field within the substation and could be used by the utilities companies to predict the field distribution following any disturbance and thus assist in the planning for the future substation refurbish or upgrade.

\section{CONCLUSION}

In this paper, a simple computational approach has been proposed for predicting magnetic field distribution within the substation environment. The method has been verified through direct comparison with test results conducted at a particular substation during working conditions and good agreement is observed. For the normal operation conditions the maximum values of the magnetic field were found to be below public exposure limits. Using the same algorithm, the magnetic field was then calculated for the cases when a lightning pulse was injected to the substation as a disturbance. The model is able to calculate the magnetic field using two different approaches, in time domain and in frequency domain. Both methods yield very close results and predict that the maximum magnetic field value exceeds the public and the occupational exposure limit set by ICNIRP. For a lightning strike in the substation, a smaller time step in the current load flow analysis is required in order to predict the peek current magnitude and to capture the fast transient so that the magnetic field could be calculated accurately. Using the frequency domain approach facilitates the understanding of how each important frequency harmonic influences the field distribution within the substation and this can be used to design more efficient EMI protection for sensitive electronic and digital equipment.

\section{ACKNOWLEDGMENT}

The layout of the substation provided by the Technical University of Cluj, Faculty of Electrotechnics, Romania.

\section{REFERENCES}

[1] Working Group C4.208, "EMC within Power Plants and Substations," CIGRE2013.

[2] U. M. Peterlin and T. Zivic, "Electromagnetic Compatibility Levels in Power Plants and Substations," IEEE International Symposium on Electromagnetic Compatibility (EMC), 2015.

[3] U. M. Peterlin, "Guidelines for Electromagnetic Compatibility Provision in Power Plants and Substations," International Symposium on Electromagnetic Compatibility (EMC Europe 2013), 2013.

[4] E. Csanyi. (2012). Smart Grid Concept and Characteristics.

[5] C. P. Nicolaou, A. P. Papadakis, P. A. Razis, G. A. Kyriacou, and J. N. Sahalos., "Measurements and Predictions of Electric and Magnetic Fields From Power Lines," Electric Power Systems Research, vol. 81, pp. 1107116, 2011.

[6] C. Munteanu, G. Visan, and I. T. Pop, "Electric and Magnetic Field Distribution Inside High Voltage Power Substations. Numerical Modeling and Experimental Measurements," IEEJ Transactions on Electrical and Electronic Engineering, vol. 5, pp. 40-45, 2010.

[7] C. Munteanu, V. Topa, I. T. Pop, A. Racasan, and E. Merdan, "Advances on the Electromagnetic Field Distribution Analysis inside High Voltage Substations," International Universities' Power Engineering Conference (UPEC 2011), 2011

[8] I. Said, H. B. Hussain, and V. Dave, "Characterization of Magnetic Field at Distribution Substations," 9th International Conference on Environment and Electrical Engineering (EEEIC), pp. 423 - 426, 2010. 
[9] W. G. Duff, Fundamentals of Electromagnetic Compatibility. Gainesville, Va.: Interference Control Technologies, 1988.

[10] W. H. Organization, "What are electromagnetic fields? - Current standards," in Exposure to extremely low frequency fields, ed. http://www.who.int/peh-emf/about/WhatisEMF/en/index4.html: WHO Media centre, 2007.

[11] F. T. Ulaby, Electromagnetics for Engineer: Peason/Prentice Hall, 2005.

[12] D. N. FÎTA, L. Muresan, C. Cheleman, and M. Grebenisan, "The Modelling of Rosiori Power Station 400/220/20 KV Retechnologized of Transelectrica Company. The Simulation of the Permanent Regime With EDSA Programme," 6TH International Conference Electromechanical dan Power Systems, 2007.

[13] W. Krajewski, "Numerical Modelling of The Electric Field in HV Substations," IEE Proceedings - Science, Measurement and Technology, vol. 151, pp. 267-272, 2004.
[14] A. P. P. Charalambos P. Nicolaou, Panos A. Razisa, George A. Kyriacou, John N. Sahalos, "Simplistic numerical methodology for magnetic field prediction in open air type substations," Electric Power Systems Research, vol. 81, pp. 2120-2126, 2011.

[15] "DIgSILENT PowerFactory 15," in User Manual, 15 ed. Gomaringen, Germany: DIgSILENT GmbH, 2015.

[16] C. P. Nicolaou, A. P. Papadakis, P. A. Razis, G. A. Kyriacou, and J. N. Sahalos, "Experimental measurement, analysis and prediction of electric and magnetic fields in open type air substations," Electric Power Systems Research, vol. 90, 2012.

[17] I. E. Commission, "IEC 62305-1 International Standard," in Protection Against Lightning, ed, 2010.

[18] R. A. D. Marcus O. Durham, "Lightning, Transient \& High Frequency Impact On Material Such As Corrugated Tubing. Frontiers of Power. ," 2008 . 